\title{
Increased production of IL-6 and IL-17 in lipopolysaccharide- stimulated peripheral mononuclears from patients with rheumatoid arthritis
}

\author{
Lucia Chovanova ${ }^{1,2}$, Miroslav Vlcek ${ }^{1,2}$, Katarina Krskova ${ }^{1}$, Adela Penesova ${ }^{1}$, Zofia Radikova ${ }^{1}$, \\ Jozef Rovensky ${ }^{3}$, Dana Cholujova ${ }^{4}$, Jan Sedlak ${ }^{4}$ and Richard Imrich ${ }^{1,2}$ \\ ${ }^{1}$ Institute of Experimental Endocrinology, Slovak Academy of Sciences, Bratislava, Slovak Republic \\ ${ }^{2}$ Center for Molecular Medicine, Slovak Academy of Sciences, Bratislava, Slovak Republic \\ ${ }^{3}$ National Institute of Rheumatic Diseases, Piestany, Slovak Republic \\ ${ }^{4}$ Cancer Research Institute, Slovak Academy of Sciences, Bratislava, Slovak Republic
}

\begin{abstract}
TLR4-mediated inflammatory responses are important for innate immune functions, thus their alterations may participate in the pathogenesis of rheumatoid arthritis (RA). Cortisol is one of the most potent immunomodulatory hormones involved in control of inflammation. In this study, we analyzed TLR4-mediated responses and cortisol effects on the process in peripheral blood mononuclear cells (PBMC) from RA patients. Lipopolysaccharide-stimulated PBMC from 23 female patients and 15 healthy controls were cultured in the presence or absence of cortisol $(1 \mu \mathrm{M})$ for $24 \mathrm{~h}$. A panel of 17 inflammatory cytokines was analyzed in the cell culture supernatants. Higher $(p<0.05)$ concentrations of IL-6, IL-17 and MCP-1 were found in lipopolysaccharide-stimulated PBMC from RA patients compared to controls. After normalization of stimulated cytokine secretion to unstimulated cells, a significantly higher $(p<0.05)$ IL- 6 and G-CSF production was found in RA PBMC. Cortisol induced stronger $(p<0.05)$ suppression of lipopolysaccharide-stimulated secretion of IL-1 $\beta$, IL-6, IL-17 and G-CSF in RA group compared to controls. The observed higher production of the key inflammatory cytokines by RA PBMC to lipopolysaccharide stimulation supports involvement of TLR4-mediated processes in RA pathogenesis. The higher sensitivity of LPS-stimulated RA PBMC to immunosuppressive effects of cortisol may reflect adaptive processes to chronic inflammation.
\end{abstract}

Key words: Inflammation - Cytokines - Cortisol - Lipopolysaccharide - Rheumatoid arthritis

Abbreviations: CRP, C-reactive protein; DAS28, disease activity score 28; GC, glucocorticoids; IFN- $\gamma$, interferon $\gamma$; IL, interleukin; LPS, lipopolysacharide; PBMC, peripheral blood mononuclear cells; RA, rheumatoid arthritis; TLRs, Toll-like receptors; TNF- $\alpha$, tumor necrosis factor $\alpha$.

\section{Introduction}

Rheumatoid arthritis (RA) is a chronic inflammatory disease characterized by persistent joint inflammation with concomitant cartilage and bone destruction. Despite

Correspondence to: Lucia Chovanova, Institute of Experimental Endocrinology, Slovak Academy of Sciences, Vlarska 3, Bratislava 833 06, Slovak Republic

E-mail: lucia.chovanova@savba.sk intensive research, many features of the immunopathology of RA are yet to be explored. Although the inflammation occurs primarily in the joint synovium in RA, peripheral blood monocytes and lymphocytes are actively recruited from the circulation to the sites of inflammation. Conversely, cytokines and chemokines released from affected tissues, modulate functional status of peripheral blood mononuclear cells (PBMC) indicating a complex involvement of the PBMC pool in the disease onset and progression. 
Several inflammatory cytokines such as tumor necrosis factor $\alpha$ (TNF- $\alpha$ ), interleukin (IL) 6, IL-1 $\beta$ and IL-17 are considered crucial in the RA pathogenesis by promoting autoimmunity, maintaining chronic inflammatory synovitis, and by driving the destruction of adjacent join tissue (Smolen and Steiner 2003). The circulating levels of cytokines not only correlate with RA activity, but their increased concentrations can be detected years before the onset of RA symptoms (Jorgensen et al. 2008). RA is characterized by a marked shift towards a T helper (Th) 1 phenotype, which has been described as proinflammatory, with overproduction of interferon $\gamma($ IFN- $\gamma$ ) and inadequate production of Th2 cytokines such as IL-4 and IL-13 (Schulze-Koops and Kalden 2001). The Th1/Th2 imbalance paradigm in RA was, however, recently refined by the identification of the Th17 and T regulatory (Treg) subsets (Boissier et al. 2008). The presence of Th17 cells in synovial fluid and peripheral blood of patients with RA suggests an involvement of this potent proinflammatory cytokine in RA pathology (Shahrara et al. 2008). Additionally, the ubiquitous expression of IL-17 receptor on fibroblasts, endothelial cells, epithelial cells and neutrophils indicates a potential of this cytokine to influence a number of effector cells and pathways involved in RA (Kolls and Linden 2004). Therefore, the cytokines join the immuno-regulatory and tissue-destructive events underlying the clinical presentation and progression of the disease. A dysregulation of the complex cytokine network, preventing normal resolution of inflammation, could be, in part, one of the major mechanisms of chronic inflammation onset and/or persistence in RA.

Several factors have been suggested to trigger local and systemic inflammatory reactions, including microbes and their products (Krause et al. 1996). Products of invading microbes may activate inflammatory cells via Toll-like receptors (TLRs), which lead to immune and inflammatory responses. TLRs expressed on monocytes, macrophages and neutrophils play a fundamental role in pathogen recognition and activation of innate immunity by production of cytokines necessary for the development of effective downstream immune responses. Recently, it has been shown that several endogenous molecules such as heat shock proteins, fragmentation products of fibronectin and hyaluronic acid can also trigger an inflammatory response via TLR2 and TLR4 (Kawai and Akira 2010). Activation of TLR4 was found to be important for the survival of pathogenic Th17 cells through induction of IL-23 production by dendritic cells suggesting an involvement in RA pathogenesis (Vanden Eijnden et al. 2006). The role of TLR4 signaling in RA synovium has been well recognized, and recently, TLR4 has been considered a target for RA therapy due to its potential to regulate a number of pathogenic cytokines including IL-17 in mouse model of arthritis (AbdollahiRoodsaz et al. 2008).
Glucocorticoids are hormones with potent immunomodulating properties, which can be regarded as antiinflammatory in supraphysiologic concentrations (approximately $1 \mu \mathrm{M})$. Supraphysiologic concentrations have been widely exploited both as a clinical therapy in RA and as a tool for understanding the mechanisms of endocrine-immune interactions (Imrich et al. 2010). Adrenal production of cortisol, the main glucocorticoid, is tightly regulated by a negative feedback loop within the hypothalamic-pituitary-adrenal (HPA) axis, and some stressors including acute systemic inflammation can activate the axis leading to increased serum cortisol concentration (Tsigos et al. 1999). Cortisol also regulates expression of several genes involved in immune response affecting production of many cytokines, chemokines, transcription factors and TLRs (Maurer et al. 2001; Galon et al. 2002; Harikai et al. 2012). It has been shown that immunomodulating properties of glucocorticoids depend on their actual concentration (Stahn et al. 2007), thus a delicate balance between cortisol at physiological conditions and during stress is crucial for an adequate control of immune response. Interestingly, in patients with chronic inflammatory diseases such as RA, adrenal function appears to be inappropriately low for given degree of inflammation (Imrich et al. 2010) suggesting either an intrinsic relative insufficiency or an adaptation to chronic inflammation at the level of the neuroendocrine system; however, the similar adaptation can be anticipated also in the immune cells.

In this study we tested the hypothesis that PBMC responses to TLR4-mediated stimulation are characterized by a specific cytokine pattern in RA patients, which may participate in the disease onset and/or persistence. Therefore, we analyzed a panel of pro- and anti-inflammatory cytokines produced by isolated PBMC before and after stimulation with lipopolysaccharide (LPS) in RA patients and matched healthy controls. The second point we were interested in, was cortisol effects on LPS-stimulated cytokine production by PBMC, which may reflect how the TLR4-mediated stimulation of PBMC is affected by glucocorticoids.

\section{Materials and Methods}

\section{Patients}

Twenty-three $(n=23)$ female patients fulfilling the 2010 ACR-EULAR classification criteria for RA were included in the study (Aletaha et al. 2010). The patients were recruited from the National Institute of Rheumatic Diseases, Piestany, Slovak Republic. Fifteen $(n=15)$ healthy female subjects with similar age and body mass index served as controls. Basic characteristics of all subjects enrolled in the study are summarized in Table 1. All the studied subjects were 
Table 1. Basic characteristics of RA patients and controls, number of patients on respective treatment, concentrations of C-reactive protein (CRP) and inflammatory cytokines in serum

\begin{tabular}{lcccc}
\hline & $\begin{array}{c}\text { controls } \\
(n=15)\end{array}$ & $\begin{array}{c}\text { RA patients } \\
(n=23)\end{array}$ & $\begin{array}{c}\text { RA GC } \\
(n=12)\end{array}$ & $\begin{array}{c}\mathrm{RA} \mathrm{GC}^{-} \\
(n=11)\end{array}$ \\
\hline Age (years) & $29.9 \pm 1.3$ & $30.5 \pm 1.2$ & $31.6 \pm 1.7$ & $29.5 \pm 1.6$ \\
Body mass index $\left(\mathrm{kg} / \mathrm{m}^{2}\right)$ & $21.6 \pm 0.5$ & $21.5 \pm 0.6$ & $21.6 \pm 0.7$ & $21.5 \pm 0.9$ \\
Disease duration (years) & - & $7.4 \pm 0.7$ & $6.5 \pm 0.5$ & $8.3 \pm 1.3$ \\
DAS28 & - & $3.4 \pm 0.2$ & $3.4 \pm 1.3$ & $3.1 \pm 0.9$ \\
Glucocorticoid treatment & - & 12 & 12 & - \\
NSAID treatment & - & 21 & 12 & 10 \\
Methotrexate treatment & - & 19 & 9 & 11 \\
CRP (mg/l) & $1.2 \pm 0.9$ & $7.4 \pm 2.9^{* * *}$ & $11.7 \pm 5.7^{* * *}$ & $3.26 \pm 1.06^{*}$ \\
IL-6 (pg/ml) & $1.4 \pm 0.5$ & $18.9 \pm 6.0^{* * *}$ & $31.12 \pm 10.82^{* * *}$ & $6.80 \pm 2.15^{* *}$ \\
IL-8 (pg/ml) & $2.23 \pm 0.8$ & $5.7 \pm 2.5^{* * *}$ & $8.60 \pm 4.96^{* * *}$ & $2.84 \pm 0.20$ \\
IFN- $\gamma(\mathrm{pg} / \mathrm{ml})$ & $2.13 \pm 1.4$ & $17.4 \pm 11.0^{*}$ & $30.97 \pm 21.60^{*}$ & $3.84 \pm 2.15$ \\
TNF- $\alpha$ (pg/ml) & $0.6 \pm 0.3$ & $13.9 \pm 11.4^{*}$ & $26.80 \pm 22.68^{* *+}$ & $1.10 \pm 0.63$ \\
\hline
\end{tabular}

Disease activity score 28 (DAS28) was calculated using number of swollen joints, number of tender joints and plasma CRP. IL, interleukin; NSAID, nonsteroidal anti-inflammatory drug; RA GC ${ }^{+}$, glucocorticoid treatment; RA GC ${ }^{-}$, without glucocorticoid treatment; TNF- $\alpha$, tumor necrosis factor $\alpha .{ }^{\star} p<0.05 ;{ }^{* *} p<0.01 ;{ }^{* *} p<0.001 \mathrm{RA} v$ s. controls; ${ }^{+} p<0.05 \mathrm{RA} \mathrm{GC}^{+} v s . \mathrm{RA} \mathrm{GC}^{-}$.

non-smokers, had negative history of endocrine disorders, diabetes or impaired glucose tolerance, age limit was 18-40 years, BMI limit was $18-25 \mathrm{~kg} / \mathrm{m}^{2}$. The last dose of the medicaments was administered 24 hours prior to the investigation. Twelve patients used low-dose glucocorticoids treatment (less than $8.5 \mathrm{mg}$ of prednisone or equivalent per day). RA activity was quantified as the disease activity score 28 (DAS28) with three variables i.e. number of swollen joints, number of tender joints and plasma C-reactive protein (CRP) concentration using an online calculator http://www.das-score.nl/dasculators.html. Eight $(n=8)$ RA patients had low (DAS28-CRP $\leq 3.2)$, fourteen $(n=14)$ patients had moderate (DAS28-CRP $>3.2$ and $\leq 5.1$ ), and one $(n=1)$ patient had high (DAS28-CRP $>5.1)$ disease activity. Patients with moderate and high disease activity were considered a single RA subgroup. All subjects gave informed written consent and the study was approved by the Ethics Committee of the National Institute of Rheumatic Diseases, Piestany, Slovakia in agreement with the ethical guidelines of the Declaration of Helsinki as revised in 2000.

\section{Cell isolation}

Whole blood from RA patients and healthy volunteers was collected in heparin-containing tubes. PBMC were isolated by density gradient centrifugation using lymphocyte separation medium (LSM 1077, PAA, Pasching, Austria) and washed three times with RPMI 1640 (Gibco (Invitrogen), Carlsbad, CA, USA). After determination of cell number in counting chamber, $\mathrm{PBMC}$ were resuspended in complete medium (RPMI 1640, 10\% Fetal Bovine Serum (Gibco
(Invitrogen), Carlsbad, CA, USA), $100 \mathrm{IU} / \mathrm{ml}$ penicillin, $100 \mu \mathrm{g} / \mathrm{ml}$ streptomycin (both Sigma-Aldrich, St. Louis, MO, USA)). Cells were cultivated in 24-well cell culture plates (Sarstedt AG \& Co, Nümbrecht, Germany) in $0.6 \mathrm{ml}$ of suspension at final concentration $2 \times 10^{5}$ cells $/ \mathrm{ml}$.

\section{Culture conditions}

Cells were kept unstimulated or were stimulated by LPS (Sigma-Aldrich, St. Louis, MO, USA) in concentration 0.5 $\mu \mathrm{g} / \mathrm{ml}$, both conditions were cultured with or without added cortisol in final concentration $1 \mu \mathrm{M}$ (Sigma-Aldrich, St. Louis, MO, USA), resulting in four different conditions. All cases were set in fourplicates. After $24 \mathrm{~h}$ of incubation at $37^{\circ} \mathrm{C} / 5 \%$ $\mathrm{CO}_{2}$ culture media were collected and centrifuged, cell-free supernatants were kept frozen at $-80^{\circ} \mathrm{C}$ until analyzed.

\section{Cytokine analysis}

For measurement of serum levels of cytokines, blood was drawn into polyethylene tubes; after clotting at room temperature for about $60 \mathrm{~min}$, the blood was centrifuged and the supernatant was stored at $-20^{\circ} \mathrm{C}$ until assayed. Levels of cytokines in culture media were measured using Bio-Plex Human Cytokine 17-plex panel kit (Bio-Rad, Hercules, CA, USA). Supernatants were analyzed simultaneously for the following 17 cytokines: IL-1 $\beta$, IL-2, IL-4, IL-5, IL-6, IL-7, IL-8, IL-10, IL-12 (p70), IL-13, IL-17, granulocyte colonystimulating factor (G-CSF), granulocyte-macrophage colony-stimulating factor (GM-CSF), interferon- $\gamma$ (IFN- $\gamma$ ), monocyte chemoattractant protein-1 (MCP-1), macrophage 
inflammatory protein-1 $\beta$ (MIP-1 $\beta$ ), and TNF- $\alpha$. Five out of 17 cytokines of the panel were excluded from the analysis because of measured levels being below (IL-2, IL-5, IL-7 and GM-CSF) or above the detection limit (MIP-1 $\beta$ ). Serum levels of IL- $1 \beta$, IL-4, IL-6, IL- 8 , IL-10, IFN- $\gamma$ and TNF- $\alpha$ were determined by multiplex bead assay (Bio-Plex Human Cytokine 8-plex panel; Bio-Rad, Hercules, CA, USA). Serum CRP was measured by immunoturbidimetric assay on automated biochemistry analyzer (Hitachi 917, Roche Diagnostics, Basel, Switzerland).

\section{Statistical analysis}

Statistical analysis of obtained data was performed using SPSS v11.5 software (SPSS Inc., Chicago, IL, USA). Measured parameters were compared by paired and unpaired T-test or Mann-Whitney test and Wilcoxon test according to data distribution. Spearman's correlation was used to analyze relations between variables. $p<0.05$ was considered statistically significant.

\section{Results}

Serum levels of cytokines in RA patients and healthy individuals

Significantly $(p<0.05)$ higher CRP, IL-6, IL-8, IFN- $\gamma$ and TNF- $\alpha$ levels were found in sera of patients with RA compared to the control group (Table 1). The levels of serum IL-6 significantly $(p<0.05)$ correlated with CRP levels in both study groups. A positive correlation between serum IL- 6 and DAS28 $(r=0.655, p<0.05)$ and between serum IFN- $\gamma$ and the presence of low-dose glucocorticoid treatment $(r=0.435, p<0.05)$ was found in RA patients. The serum levels of IL- 6 correlated with serum levels of IFN- $\gamma$ $(\mathrm{r}=0.650, p<0.05)$ and TNF- $\alpha(\mathrm{r}=0.542, p<0.05)$ in patients but not controls (Fig. 1).

\section{LPS-stimulated cytokine production}

The only observed difference between cytokine secretion by unstimulated PBMC in RA patients compared to controls was the significantly $(p<0.05)$ higher concentration of IL-12 (10.4 vs. $8.1 \mathrm{pg} / \mathrm{ml}$, respectively) and MCP-1 (791.5 vs. $488.2 \mathrm{pg} / \mathrm{ml}$, respectively). The addition of LPS to cell culture medium significantly $(p<0.05)$ increased production of IL- $1 \beta$, IL-6, IL-10, IL-17, IFN- $\gamma$, TNF- $\alpha$, G-CSF and IL- 4 cytokines, but not IL- 8 , IL-12 and IL-13 in RA patients. In control group, a significant $(p<0.05)$ increase of IL- $1 \beta$, IL- 10 , IL-17, IFN- $\gamma$, TNF- $\alpha$ and IL-4, but not IL-6, IL-8, IL-12, IL-13 and G-CSF was observed. A significantly $(p<0.05)$ higher concentrations of IL-6, IL-17 and MCP-1 were found in LPS-stimulated PBMC from RA patients compared to those from healthy controls (Table 2). When LPS-stimulated cytokine concentrations were normalized to unstimulated ones, a significantly $(p<0.05)$ greater increase in IL- 6 and G-CSF levels were found in RA patients than in healthy subjects.

\section{Cortisol effects on cytokine production}

The addition of cortisol to unstimulated PBMC caused a significant decrease $(p<0.05)$ in levels of IL-1 $\beta$, IL-6, IL10 , IL-17, IFN- $\gamma$, TNF- $\alpha$ and IL- 4 in both groups, with the exception of IL-13, G-CSF and MCP-1 in RA patients and IL-8, IL-12, IL-13, G-CSF and MCP-1 in controls (Fig. 2). In LPS-stimulated cells, the presence of cortisol suppressed the production of IL-1 $\beta$, IL-6, IL-10, IL-12, IL-17, IFN- $\gamma$, TNF- $\alpha$
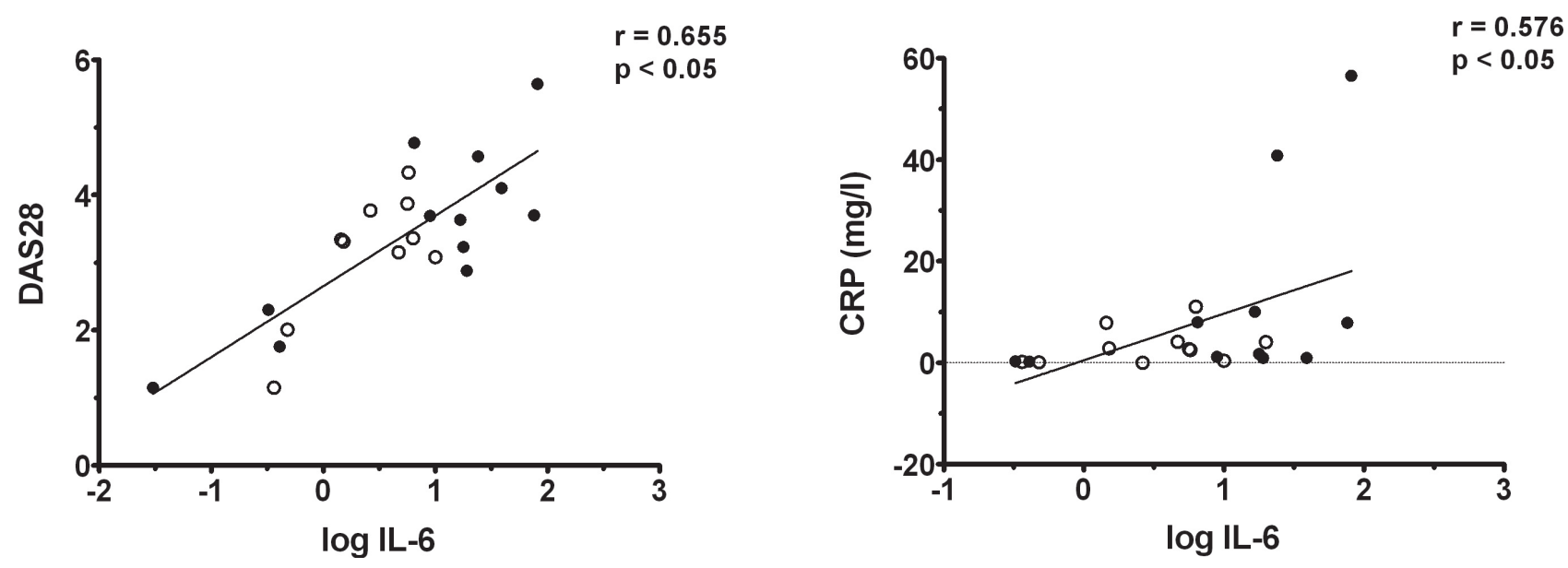

Figure 1. The correlation between baseline serum IL-6 levels and DAS28 and CRP. The logarytmic transformation of serum cytokine levels was used. Open circles refer to $\mathrm{GC}^{-}$patients; closed circles refer to $\mathrm{GC}^{+}$patients. r, correlation coefficient. 
Table 2. Production of cytokines IL-6, IL-17 and MCP-1 in the absence and presence of LPS in RA patients and controls

\begin{tabular}{lccc}
\hline Cytokines & Baseline & Stimulation & $\begin{array}{c}\text { Mean differences } \\
\text { after stimulation (\%) }\end{array}$ \\
\hline IL-6 (pg/ml) & & & $-224.6(-2.8)$ \\
CN & $7936.7 \pm 984.2$ & $7712.1 \pm 931$ & $2662.8(29.9)$ \\
RA & $8887.5 \pm 1290.7$ & $11550.3 \pm 1339.6^{*+}$ & $2887.4(303.7)$ \\
RA-CN difference (Units) & 950.8 & 3838.2 & 37.9 \\
RA-CN difference (\%) & 11.9 & 49.8 & $2.0(10.6)$ \\
\hline IL-17 (pg/ml) & $18.9 \pm 0.63$ & $20.9 \pm 1.04^{+}$ & $3.6(14.4)$ \\
CN & $20.3 \pm 0.62$ & $23.9 \pm 0.82^{*+}$ & $1.6(14.3)$ \\
RA & 1.4 & 3.0 & 7.0 \\
RA-CN difference (Units) & 7.4 & 14.4 & $-256.7(-52.6)$ \\
RA-CN difference (\%) & & & $-384.0(-48.5)$ \\
\hline MCP-1 (pg/ml) & $488.2 \pm 66.5$ & $231.5 \pm 27.4^{+}$ & $-127.3(-41.9)$ \\
CN & $791.5 \pm 84.4^{*}$ & $407.5 \pm 38.6^{*+}$ & 13.9 \\
RA & 303.3 & 176.0 & 76.0 \\
RA-CN difference (Units) & 62.1 & & \\
RA-CN difference (\%) & & \\
\hline
\end{tabular}

Data are expressed as a concentration of particular cytokine in culture media \pm SE. ${ }^{\star} p<0.05 \mathrm{RA} v s$. Controls; ${ }^{+} p<0.05 \mathrm{LPS}$ stimulated $v s$. baseline level. CN, control; IL, interleukin; LPS, lipopolysacharide; MCP, monocyte chemoattractant protein; RA, rheumatoid arthritis.

and IL-4 cytokines, but not IL-8, IL-13, MCP-1 and G-CSF in both groups. The levels of G-CSF increased significantly $(p<0.05)$ after addition of cortisol to unstimulated cells from RA patients and controls. Cortisol induced significantly $(p<$ 0.05 ) stronger suppression of LPS-stimulated secretion of IL-1 $\beta$, IL-6, IL-17 and G-CSF in RA patient compared to controls.

$R A$ activity and glucocorticoid treatment effects on cytokine production

There was no significant difference between patients with low and moderate disease activity in the concentrations of cytokines produced by PBMC with respect to presence/absence of LPS or the presence/absence of cortisol. A negative correlation was found between DAS28 and cortisol-induced suppression of IL-1 $\beta$ ( $\mathrm{r}=0.423, p<0.05)$, IL-4 $(\mathrm{r}=0.651$, $p<0.05)$, IFN- $\gamma(\mathrm{r}=0.517, p<0.05)$ and G-CSF $(\mathrm{r}=0.438$, $p<0.05$ ) secretion by LPS-stimulated PBMC (Fig. 3 ). We did not find any difference in cytokine responses to LPS stimulation or cortisol suppression in relation to the lowdose glucocorticoid treatment in RA patients.

\section{Discussion}

The imbalance between pro- and anti-inflammatory cytokines is a key concept in initiation and progression of RA (McInnes and Schett 2007). In our study, we ana-
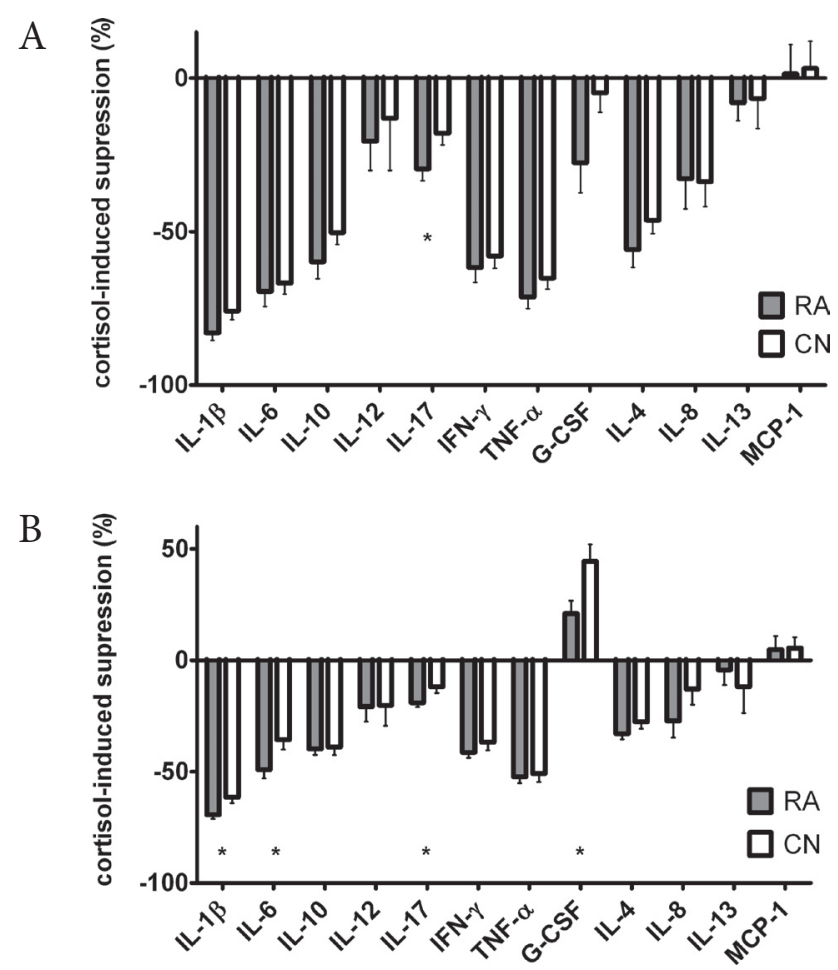

Figure 2. The effect of cortisol on cytokine production by unstimulated (A) and LPS-stimulated (B) PBMC. Data are expressed as a percentage of (A) basal production and (B) LPS-induced cytokine production represented by solid reference lines. Bars represent cytokine levels produced in the presence of cortisol in control group (CN; white bars) and RA patients (RA; grey bars); ${ }^{\star} p<0.05$. 
A

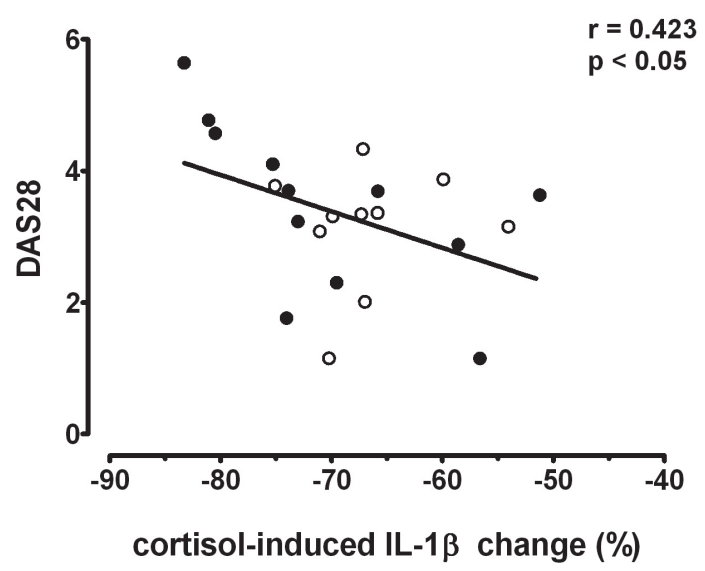

$\mathrm{C}$

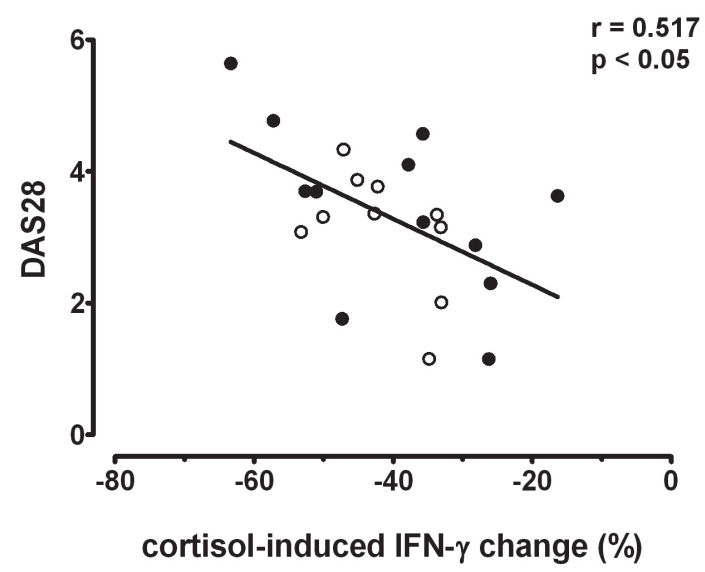

B

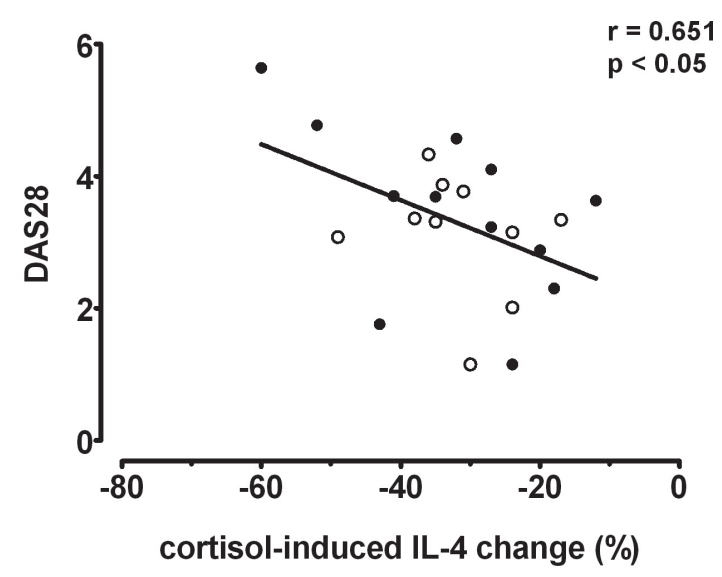

$\mathrm{D}$

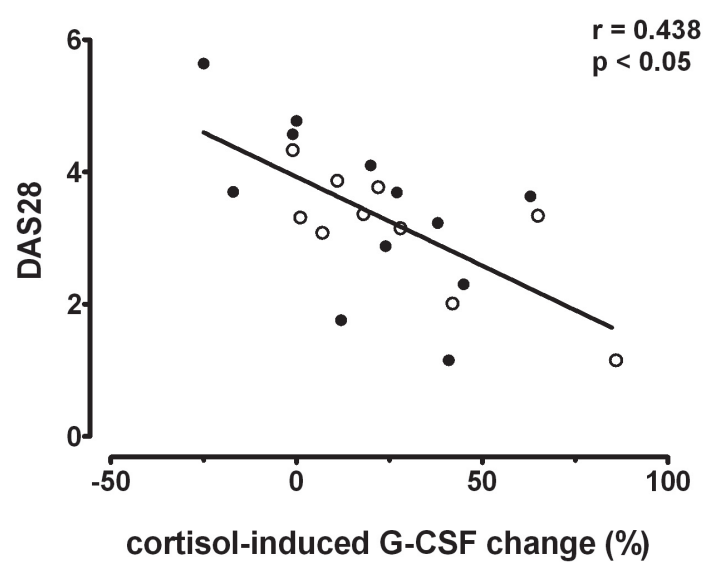

Figure 3. The correlation between DAS28 and the change of production of IL-1 1 (A), IL-4 (B), IFN- $\gamma$ (C) and G-CSF (D) by LPS-stimulated PBMC from RA patients induced by cortisol. Open circles refer to $\mathrm{GC}^{-}$patients with glucocorticoid therapy; closed circles refer to $\mathrm{GC}^{+}$patients without glucocorticoid therapy; $\mathrm{Y}$ axis represents DAS28 values, $\mathrm{X}$ axis represents the percentage of change in cytokine production induced by cortisol. r, correlation coefficient.

lyzed a pattern of cytokines production by PBMC from RA patients in response to LPS stimulation. The main finding of our study is that LPS-stimulated PBMC from RA patients produced significantly more IL-6, IL-17 and MCP-1 (Fig. 1). An increase of IL-6 secretion by PBMC after LPS stimulation was reported previously (Kowalski et al. 2008). Also, an increased production of IL-17 by LPS-stimulated whole blood cultures from RA patients has been reported recently (Zivojinovic et al. 2012). A dysregulation or overproduction of IL- 6 has been recognized as one of the hallmarks of several autoimmune diseases including RA (Kimura and Kishimoto 2010). Also, IL-17 producing Th17 cells are considered one of the key players in the RA pathogenesis. Both cytokines are functionally closely interlinked, since IL-6 together with transforming growth factor beta (TGF- $\beta$ ) induces the development of Th17 cells from naive T cells. On the other hand, IL-6 inhibits TGF- $\beta$-induced Treg differentiation (Bettelli et al. 2006). Thus, our finding provides important evidence supporting the concept of IL-6/IL-17 involvement in RA pathogenesis in the setting of TLR4mediated immune functions.

The design of our study did not allow us to determine whether or not the observed increased cytokine response to LPS-stimulation reflects an upregulated secretion by a specific PBMC subset. However, we can assume a substantial contribution of Th17 cells into the total measurable IL-17 in our experiment. Alternatively, a higher proportion of IL17 producing cells in RA PBMC can explain our findings. Data about the percentage of circulating Th17 cells in RA are 
inconsistent showing unchanged (Yamada et al. 2008) and increased numbers of Th17 cells (Chen et al. 2012; Samson et al. 2012).

LPS is a TLR4 ligand and probably the most frequent stimulus of the innate immune system (Sabroe et al. 2002). During cell contact with LPS, TLR4 interacts with CD14 forming a receptor complex, and activates second messenger and signal transduction pathways. As a result, LPS triggers a wide variety of cellular responses, including the production of cytokines and chemokines (Yang et al. 1998). RA synoviocytes were found to secrete IL-6 and metalloproteinases in response to LPS, supporting a strong proiflammatory and joint destructing role of TLR4 signaling (Brentano et al. 2009). TLR3 and TLR4 were found to be overexpressed in RA synovial fibroblasts, which can result in persistent inflammation and joint destruction in RA (Ospelt et al. 2008). Implications of TLR4 signaling for the PBMC pool is, however, less clear with regard to RA pathogenesis. PBMC from patients with newly diagnosed RA spontaneously generated significantly smaller amounts of IL-6 and TNF- $\alpha$ as compared to monocytes from osteoarthritis patients or healthy subjects and stimulation with LPS resulted in dramatically higher augmentation of cytokine production in cells from RA patients as compared to the other two groups (Kowalski et al. 2008). The observed higher responsiveness of monocytes from RA subjects to LPS as compared to healthy controls in our study could be explained by increased expression of TLR4 receptors on cell surface as found by other authors (Huang et al. 2007). Although the TLR4 detection on cell surface was not included in our experiments, we suggest that presented results support the putative role of TLR4 receptors in the development of inflammatory responses and that circulating inflammatory cells exhibit higher tendency to generation of proinflammatory cytokines after TLR4 stimulation in RA.

In order to overcome relatively high interindividual variability in LPS-stimulated cytokine production, we also analyzed the responses in relation to LPS-untreated cells. Our results showed that PBMC from RA patients responded to LPS with a higher production of IL- 6 and G-CSF indicating that the latter cytokine can also be involved in altered LPSmediated immune responses in RA.

Surprisingly, we did not find any differences in proportion of Th1 and Th 2 cytokines in our study. IFN- $\gamma$ is mainly secreted by Th 1 cells, whereas Th 2 cells generally produce IL-4 and IL-10. Several studies have reported a preferential activation of Th1 cells in rheumatoid synovium, suggesting that Th1 rather than Th2 cytokines are involved in the pathogenesis of the disease (Dolhain et al. 1996; Park et al. 2001). Our results, however, do not support the proposed shift to Th1 response in PBMC after stimulation with LPS. Further studies of TLR-mediated immune function in RA are required to analyze precise mechanisms of the changes in cytokine pattern observed in our experiments.

DAS28 is a clinically important proxy for actual inflammatory status in RA. Our present results indicate that RA activity, as measured by CRP-derived DAS28, can have a significant impact on ex vivo PBMC responses (Fig. 3). A positive correlation between DAS28 and serum IL-17 synovial fluid levels was reported by other authors (Chen et al. 2012; Rosu et al. 2012). The decrease in DAS28 was also associated with decrease in the percentage of Th17 cells and increased percentage of Tregs after treatment with tocilizumab, which is in line with the potential role of this PBMC subset in pathogenesis of RA (Samson et al. 2012). In the present study, we also attempted to correlate serum concentrations of specific cytokines with their production ex vivo by PBMC. Despite the fact that RA patients had significantly elevated levels of proinflammatory IL-6, IL-8, IFN- $\gamma$ and TNF- $\alpha$ in serum as anticipated during chronic inflammation (Hueber et al. 2007; Chen et al. 2011; Chung et al. 2011), we did not find any relationship between the serum levels and ex vivo production.

The second aim of this study was to evaluate the immunomodulatory effect of cortisol on cytokine production by PBMC. Immune processes during inflammatory response are regulated by many intrinsic factors including stress hormones such as cortisol, affecting many immune processes e.g. T cell differentiation, chemokine and antibody production. One of the major effects of cortisol appears to be on differentiation of Th cells, shifting the immune response towards Th2 cytokine profile (Daynes and Araneo 1989; Moynihan et al. 1998). The dose of cortisol used in our experiments was comparable to those achieved during intense stress response. Similar concentrations were used previously to demonstrate immunomodulatory effects of corticosteroids in vitro (Uchakin et al. 2002). In the present study, we observed a stronger suppression of IL-1 $\beta$, IL-6, IL-17, and G-CSF production by RA cells compared to those from controls (Fig. 2). Moreover, degree of IL-1, IL4 , IFN- $\gamma$ and G-CSF suppression correlated with DAS28, meaning greater suppression in patients with higher disease activity (Fig. 3). These results indicate a tendency to increased sensitivity of RA PBMC to cytokine-suppressive effects of cortisol. A possible explanation for the findings could be an adaptation to persistent inflammation at the level of immune cells in RA, based on previous findings of our group and others showing a subtle HPA dysfunction in RA. However, cortisol secretion appears to be intact in RA patients (Straub et al. 2002; Rovensky et al. 2004; Eijsbouts et al. 2005; Imrich and Rovensky 2010). An altered expression of glucocorticoid receptors or regulation of other components of the related downstream signaling pathways could also be considered. 
Our study has several limitations; first, the cytokine panel analysis did not allow us to determine which cell subset overproduced the particular cytokine. The cell subset analysis would be very informative especially considering IL-17 involvement in RA pathogenesis. Second, the TLR4 and GR receptor expressions would provide better understanding of LPS and cortisol actions in the present setting. These questions deserve to be addressed in future studies.

In conclusion, our study demonstrates higher responses of key cytokines such as IL-6 and IL-17 from RA PBMC in response to TLR4-mediated stimulation. LPS-stimulated PBMC from more active RA patients had higher sensitivity to suppressive effects of cortisol suggesting a presence of adaptive changes to chronic inflammation in the immune system.

Acknowledgements. This work was supported by the grants VEGA 2/0084/12, VEGA 2/0018/12 and N00024 RASGENAS.

Conflict of interest: The authors have declared that there is no conflict of interest.

\section{References}

Abdollahi-Roodsaz S., Joosten L. A., Koenders M. I., Devesa I., Roelofs M. F., Radstake T. R., Heuvelmans-Jacobs M., Akira S., Nicklin M. J., Ribeiro-Dias F., van den Berg W. B. (2008): Stimulation of TLR2 and TLR4 differentially skews the balance of $\mathrm{T}$ cells in a mouse model of arthritis. J. Clin. Invest. 118, 205-216

http://dx.doi.org/10.1172/JCI32639

Aletaha D., Neogi T., Silman A. J., Funovits J., Felson D. T., Bingham C. O., 3rd, Birnbaum N. S., Burmester G. R., Bykerk V. P., Cohen M. D. et al. (2010): 2010 Rheumatoid arthritis classification criteria: an American College of Rheumatology/European League Against Rheumatism collaborative initiative. Arthritis Rheum. 62, 2569-2581 http://dx.doi.org/10.1002/art.27584

Bettelli E., Carrier Y., Gao W., Korn T., Strom T. B., Oukka M., Weiner H. L., Kuchroo V. K. (2006): Reciprocal developmental pathways for the generation of pathogenic effector TH17 and regulatory T cells. Nature 441, 235-238 http://dx.doi.org/10.1038/nature04753

Boissier M. C., Assier E., Falgarone G., Bessis N. (2008): Shifting the imbalance from Th1/Th2 to Th17/treg: the changing rheumatoid arthritis paradigm. Joint Bone Spine. 75, 373-375 http://dx.doi.org/10.1016/j.jbspin.2008.04.005

Brentano F., Kyburz D., Gay S. (2009): Toll-like receptors and rheumatoid arthritis. Methods. Mol. Biol. 517, 329-343 http://dx.doi.org/10.1007/978-1-59745-541-1_20

Daynes R. A., Araneo B. A. (1989): Contrasting effects of glucocorticoids on the capacity of $\mathrm{T}$ cells to produce the growth factors interleukin 2 and interleukin 4. Eur. J. Immunol. 19, 2319-2325

http://dx.doi.org/10.1002/eji.1830191221
Dolhain R. J., van der Heiden A. N., ter Haar N. T., Breedveld F. C., Miltenburg A. M. (1996): Shift toward T lymphocytes with a T helper 1 cytokine-secretion profile in the joints of patients with rheumatoid arthritis. Arthritis Rheum. 39, 1961-1969 http://dx.doi.org/10.1002/art.1780391204

Eijsbouts A. M., van den Hoogen F. H., Laan R. F., Hermus A. R., Sweep C. G.,van de Putte L. B. (2005): Hypothalamic-pituitaryadrenal axis activity in patients with rheumatoid arthritis. Clin. Exp. Rheumatol. 23, 658-664

Galon J., Franchimont D., Hiroi N., Frey G., Boettner A., EhrhartBornstein M., O'Shea J. J., Chrousos G. P., Bornstein S. R. (2002): Gene profiling reveals unknown enhancing and suppressive actions of glucocorticoids on immune cells. FASEB J. 16, 61-71 http://dx.doi.org/10.1096/fj.01-0245com

Harikai N. (2012): Characteristic induction of steroidogenic factor 1 (SF-1) and DAX-1 and enhanced expression of glucocorticoid synthesis-related genes in adrenals from spontaneously hypertensive rats. Gen. Physiol. Biophys. 31, 39-45 http://dx.doi.org/10.4149/gpb_2012_004

Huang Q., Ma Y., Adebayo A., Pope R. M. (2007): Increased macrophage activation mediated through toll-like receptors in rheumatoid arthritis. Arthritis Rheum. 56, 2192-2201 http://dx.doi.org/10.1002/art.22707

Hueber W., Tomooka B. H., Zhao X., Kidd B. A., Drijfhout J. W., Fries J. F., van Venrooij W. J., Metzger A. L., Genovese M. C., Robinson W. H. (2007): Proteomic analysis of secreted proteins in early rheumatoid arthritis: anti-citrulline autoreactivity is associated with up regulation of proinflammatory cytokines. Ann. Rheum. Dis. 66, 712-719 http://dx.doi.org/10.1136/ard.2006.054924

Chen D. Y., Chen Y. M., Chen H. H., Hsieh C. W., Lin C. C., Lan J. L. (2011): Increasing levels of circulating Th17 cells and interleukin-17 in rheumatoid arthritis patients with an inadequate response to anti-TNF-alpha therapy. Arthritis Res. Ther. 13, R126 http://dx.doi.org/10.1186/ar3431

Chen J., Li J., Gao H., Wang C., Luo J., Lv Z., Li X. (2012): Comprehensive evaluation of different T-helper cell subsets differentiation and function in rheumatoid arthritis. J. Biomed. Biotechnol. 2012, 535361 http://dx.doi.org/10.1155/2012/535361

Chung S. J., Kwon Y. J., Park M. C., Park Y. B., Lee S. K. (2011): The correlation between increased serum concentrations of interleukin-6 family cytokines and disease activity in rheumatoid arthritis patients. Yonsei. Med. J. 52, 113-120 http://dx.doi.org/10.3349/ymj.2011.52.1.113

Imrich R., Rovensky J. (2010): Hypothalamic-pituitary-adrenal axis in rheumatoid arthritis. Rheum. Dis. Clin. North. Am. 36, 721-727 http://dx.doi.org/10.1016/j.rdc.2010.09.003

Imrich R., Vlcek M., Aldag J. C., Kerlik J., Radikova Z., Rovensky J., Vigas M., Masi A. T. (2010): An endocrinologist's view on relative adrenocortical insufficiency in rheumatoid arthritis. Ann. N. Y. Acad. Sci. 1193, 134-138 http://dx.doi.org/10.1111/j.1749-6632.2009.05362.x 
Jorgensen K. T., Wiik A., Pedersen M., Hedegaard C. J., Vestergaard B. F., Gislefoss R. E., Kvien T. K., Wohlfahrt J., Bendtzen K., Frisch M. (2008): Cytokines, autoantibodies and viral antibodies in premorbid and postdiagnostic sera from patients with rheumatoid arthritis: case-control study nested in a cohort of Norwegian blood donors. Ann. Rheum. Dis. 67, 860-866

http://dx.doi.org/10.1136/ard.2007.073825

Kawai T., Akira S. The role of pattern-recognition receptors in innate immunity: update on Toll-like receptors. Nat. Immunol. 11, 373-384 http://dx.doi.org/10.1038/ni.1863

Kimura A., Kishimoto T. (2010): IL-6: regulator of Treg/Th17 balance. Eur. J. Immunol. 40, 1830-1835 http://dx.doi.org/10.1002/eji.201040391

Kolls J. K., Linden A. (2004): Interleukin-17 family members and inflammation. Immunity 21, 467-476 http://dx.doi.org/10.1016/j.immuni.2004.08.018

Kowalski M. L., Wolska A., Grzegorczyk J., Hilt J., Jarzebska M., Drobniewski M., Synder M., Kurowski M. (2008): Increased responsiveness to toll-like receptor 4 stimulation in peripheral blood mononuclear cells from patients with recent onset rheumatoid arthritis. Mediators. Inflamm. 2008, 132732 http://dx.doi.org/10.1155/2008/132732

Krause A., Kamradt T., Burmester G. R. (1996): Potential infectious agents in the induction of arthritides. Curr. Opin. Rheumatol. 8, 203-209 http://dx.doi.org/10.1097/00002281-199605000-00007

Maurer M., Trajanoski Z., Frey G., Hiroi N., Galon J., Willenberg H. S., Gold P. W., Chrousos G. P., Scherbaum W. A., Bornstein S. R. (2001): Differential gene expression profile of glucocorticoids, testosterone, and dehydroepiandrosterone in human cells. Horm. Metab. Res. 33, 691-695 http://dx.doi.org/10.1055/s-2001-19142

McInnes I. B., Schett G. (2007): Cytokines in the pathogenesis of rheumatoid arthritis. Nat. Rev. Immunol. 7, 429-442 http://dx.doi.org/10.1038/nri2094

Moynihan J. A., Callahan T. A., Kelley S. P., Campbell L. M. (1998): Adrenal hormone modulation of type 1 and type 2 cytokine production by spleen cells: dexamethasone and dehydroepiandrosterone suppress interleukin-2, interleukin-4, and interferon-gamma production in vitro. Cell. Immunol. 184, 58-64 http://dx.doi.org/10.1006/cimm.1998.1259

Ospelt C., Brentano F., Rengel Y., Stanczyk J., Kolling C., Tak P. P., Gay R. E., Gay S., Kyburz D. (2008): Overexpression of toll-like receptors 3 and 4 in synovial tissue from patients with early rheumatoid arthritis: toll-like receptor expression in early and longstanding arthritis. Arthritis Rheum. $\mathbf{5 8}, 3684-3692$ http://dx.doi.org/10.1002/art.24140

Park S. H., Min D. J., Cho M. L., Kim W. U., Youn J., Park W., Cho C. S., Kim H. Y. (2001): Shift toward T helper 1 cytokines by type II collagen-reactive $\mathrm{T}$ cells in patients with rheumatoid arthritis. Arthritis Rheum. 44, 561-569

http://dx.doi.org/10.1002/1529-0131(200103)44:3<561::AID-ANR104>3.0.CO;2-Z

Rosu A., Margaritescu C., Stepan A., Musetescu A., Ene M. (2012): IL-17 patterns in synovium, serum and synovial fluid from treatment-naive, early rheumatoid arthritis patients. Rom. J. Morphol. Embryol. 53, 73-80

Rovensky J., Radikova Z., Imrich R., Greguska O., Vigas M., Macho L. (2004): Gonadal and adrenal steroid hormones in plasma and synovial fluid of patients with rheumatoid arthritis. Endocr. Regul. 38, 143-149

Sabroe I., Jones E. C., Usher L. R., Whyte M. K., Dower S. K. (2002): Toll-like receptor (TLR) 2 and TLR4 in human peripheral blood granulocytes: a critical role for monocytes in leukocyte lipopolysaccharide responses. J. Immunol. 168, 4701-4710

Samson M., Audia S., Janikashvili N., Ciudad M., Trad M., Fraszczak J., Ornetti P., Maillefert J. F., Miossec P., Bonnotte B. (2012): Brief report: inhibition of interleukin-6 function corrects Th17/Treg cell imbalance in patients with rheumatoid arthritis. Arthritis. Rheum. 64, 2499-2503 http://dx.doi.org/10.1002/art.34477

Shahrara S., Huang Q., Mandelin A. M., 2nd, Pope R. M. (2008): TH-17 cells in rheumatoid arthritis. Arthritis Res. Ther. 10, R93

http://dx.doi.org/10.1186/ar2477

Schulze-Koops H., Kalden J. R. (2001): The balance of Th1/Th2 cytokines in rheumatoid arthritis. Best. Pract. Res. Clin. Rheumatol. 15, 677-691 http://dx.doi.org/10.1053/berh.2001.0187

Smolen J. S., Steiner G. (2003): Therapeutic strategies for rheumatoid arthritis. Nat. Rev. Drug. Discov. 2, 473-488 http://dx.doi.org/10.1038/nrd1109

Stahn C., Lowenberg M., Hommes D. W., Buttgereit F. (2007): Molecular mechanisms of glucocorticoid action and selective glucocorticoid receptor agonists. Mol. Cell. Endocrinol. 275, $71-78$ http://dx.doi.org/10.1016/j.mce.2007.05.019

Straub R. H., Paimela L., Peltomaa R., Scholmerich J., LeirisaloRepo M. (2002): Inadequately low serum levels of steroid hormones in relation to interleukin- 6 and tumor necrosis factor in untreated patients with early rheumatoid arthritis and reactive arthritis. Arthritis Rheum. 46, 654-662 http://dx.doi.org/10.1002/art.10177

Tsigos C., Papanicolaou D. A., Kyrou I., Raptis S. A., Chrousos G. P. (1999): Dose-dependent effects of recombinant human interleukin- 6 on the pituitary-testicular axis. J. Interferon. Cytokine. Res. 19, 1271-1276 http://dx.doi.org/10.1089/107999099312948

Uchakin P. N., Tobin B. W., Morukov B. V., Larina I. V., Cubbage M. L. (2002): Type 1 vs. type 2 cytokine secretion in vitro and its regulation by hydrocortisone in humans subjected to 120-day anti-orthostatic bed-rest regime. J. Gravit. Physiol. 9, 71-82

Vanden Eijnden S., Goriely S., De Wit D., Goldman M., Willems F. (2006): Preferential production of the IL-12(p40)/IL-23(p19) heterodimer by dendritic cells from human newborns. Eur. J. Immunol. 36, 21-26 http://dx.doi.org/10.1002/eji.200535467

Yamada H., Nakashima Y., Okazaki K., Mawatari T., Fukushi J. I., Kaibara N., Hori A., Iwamoto Y., Yoshikai Y. (2008): Th1 but not Th17 cells predominate in the joints of patients with rheumatoid arthritis. Ann. Rheum. Dis. 67, 1299-1304 http://dx.doi.org/10.1136/ard.2007.080341 
Yang R. B., Mark M. R., Gray A., Huang A., Xie M. H., Zhang M., Goddard A., Wood W. I., Gurney A. L., Godowski P. J. (1998): Toll-like receptor-2 mediates lipopolysaccharide-induced cellular signalling. Nature 395, 284-288 http://dx.doi.org/10.1038/26239

Zivojinovic S., Pejnovic N., Sefik-Bukilica M., Kovacevic L., Soldatovic I., Bugarski D., Mojsilovic S., Damjanov N. (2012): Effects of TNF inhibitor on innate inflammatory and Th17 cytokines in stimulated whole blood from rheumatoid arthritis patients. Inflammopharmacology 20, 323-330

http://dx.doi.org/10.1007/s10787-012-0143-7

Received: March 11, 2013

Final version accepted: April 29, 2013 